\title{
Nonlinear Generation of Ultra-flat broadened Spectrum Based on Adaptive Pulse Shaping
}

\author{
Xin Yang, David J. Richardson, and Periklis Petropoulos \\ Optoelectronic Research Centre, University of Southampton, Highfield, Southampton, United Kingdom, SO17 1BJ \\ xy1v07@orc.soton.ac.uk
}

\begin{abstract}
We report a technique to nonlinearly generate precise spectral shapes in an adaptable and power-efficient fashion. The generation of a spectrum with a 7-nm $0.5-\mathrm{dB}$ bandwidth is demonstrated to prove the validity of the approach.

OCIS codes: (060.4370) Nonlinear Optics, Fibres; (060.5530) Pulse Propagation and Temporal Solitons
\end{abstract}

\section{Introduction}

A number of technologies have been developed that allow the phase and amplitude manipulation of optical pulse spectra thereby enabling precise shaping of pulses in a linear fashion [1-3]. Among them fibre-pigtailed pulse shapers based on liquid crystal on silicon technology (an already commercially available technology) [3] combine a high spectral resolution of a few tens of picometers with acceptable insertion losses $(\sim 5 \mathrm{~dB})$ and the possibility to dynamically change the filtering transfer characteristic on microsecond timescale [1]. On the other hand, recent developments in optical fibre technology have allowed significant advances in the nonlinear generation and tailoring of broadband spectra. When it comes to controlling the characteristics of the nonlinearly broadened spectra, extensive work has focused on the optimization of the properties of the nonlinear optical fibres [4]. The combination of linear filtering with nonlinear spectral broadening can however relax the requirements on the characteristics of the nonlinear fibre. Nonlinear spectral broadening in fibres has already been combined with linear spectral shaping at the system output to demonstrate the generation of flat supercontinua [5]. However, the inclusion of a pulse shaper before the nonlinear element allows for a more power efficient process, and even provides the potential for the manipulation of optical spectra with a spectral range that exceeds the operating bandwidth of the pulse shaper [6].

For a nonlinear element (e.g. a highly nonlinear fibre - HNLF) with given characteristics, it is possible to calculate the waveform required at the input of the system in order to obtain a target spectrum at its output. This can be achieved using backward propagation calculations based on the inverse split-step Fourier method (ISSFM) [6, 7]. However, this technique requires a precise knowledge of all the system parameters and control over the shaping function. Therefore, adaptive systems exploiting evolutionary algorithms and one or more effective feedback signals have previously been adopted in pulse shaping systems to generate the target pulse form, mainly for applications in ultrafast optics $[8,9]$. Recently, genetic algorithms were used to improve the quality of Gaussian pulses and thereby optimise the characteristics of their self-phase modulation (SPM)-induced broadened spectrum [10].

In this work, we combine Kerr nonlinearities in an HNLF with a programmable phase and amplitude filter, which we use as a pulse shaper, in order to achieve the flexible generation of pulses with user-definable spectra. We apply our technique to the generation of ultra-flat broadband spectra starting from transform limited pulses derived from $10 \mathrm{GHz}$ mode-locked laser (MLL). Previous demonstrations have achieved broadened spectra with a maximum peak-to-trough ripple of $1.5 \mathrm{~dB}$ (with a fibre Bragg grating pulse shaper [6]) and more recently $3 \mathrm{~dB}$ (when a programmable pulse shaper was used [7] - see Fig.3b). In this paper, we employ adaptive pulse shaping which allows us to generate a $7-\mathrm{nm}$ spectrum with a ripple of just $0.5 \mathrm{~dB}$. We believe that by scaling to higher power levels significantly broader spectra should also be achievable.

\section{Methodology}

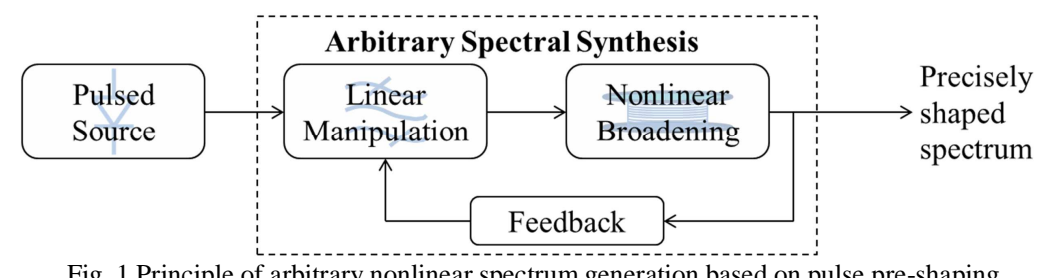

Fig. 1 Principle of arbitrary nonlinear spectrum generation based on pulse pre-shaping.

Our arbitrary spectral synthesis system comprises a programmable high resolution phase and amplitude filter (linear manipulation stage), followed by the nonlinear broadening stage (typically an amplifier and a HNLF) - see Fig.1. The spectral shaping process starts with the definition of the target signal, a third-order super-Gaussian spectrum in this case. We then use the ISSFM to calculate the waveform required at the input of the HNLF [6, 7]. Prior 
knowledge of the pulse characteristics generated by the source allows us to calculate the transfer function which needs to be fed to the programmable filter. This process assumes that (a) the exact characteristics of both the original pulses (in both phase and intensity) and the HNLF are known, (b) the amplifier prior to the HNLF is strictly linear and (c) the phase and amplitude characteristics of the filter are accurately calibrated.

In order to overcome the limitations imposed by these assumptions, we employ adaptive pulse shaping which monitors the spectrum $S_{\text {meas }}$ at the output of the system and adjusts the phase profile of the programmable filter with the aim of minimising the misfit function $M F$ between $S_{\text {meas }}$ and the target spectrum $S_{\text {target }}$ :

$$
M F^{2}=\int\left(S_{\text {meas }}-S_{\text {target }}\right)^{2} d \omega / \int S_{\text {meas }}^{2} d \omega .
$$

We have chosen to act only on the phase profile of the transfer function, since the amplitude spectrum of the shaped signal can be accurately monitored with a spectrum analyser. A spectral phase perturbation represented by a weighted summation of first kind Chebyshev polynomials of $20^{\text {th }}$ order is added to the phase profile obtained from theoretical calculations. The use of Chebyshev polynomials has an advantage that when the weights are the same for all the terms, their contribution to the entire summation is comparable. The 21 weights constitute a candidate in the search space of a two-membered evolution strategy (ES) algorithm [11]. This algorithm allows the search procedure to be performed in an area around the starting candidate in the search space. As we believed that the optimum phase profile is close to the calculated phase profile from the ISSFM, this algorithm is well suited for our application (Note that other algorithms exist which allow optimisation over a broader search space if needed).

The initial parent candidate for the ES algorithm is an array of zero weights. This enables the algorithm to evaluate the calculated phase profile from ISSFM at the beginning of the process. For the next iteration, an offspring candidate is generated by adding an array of weights which is Gaussian distributed over the parent candidate. As a result, the search area is in the vicinity of the theoretical phase profile. The algorithm is terminated when the value of $M F$ is below a pre-determined threshold, which indicates an acceptable agreement between the target and generated spectra.

\section{Experimental Results}

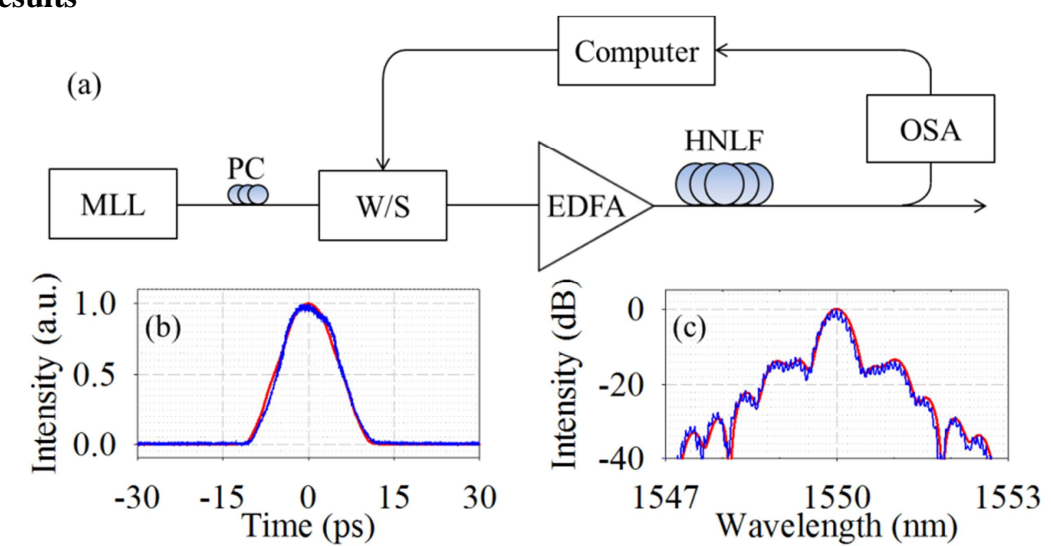

Fig. 2 Experimental setup and characteristics of the shaped pulses. (a) Experimental setup. (b) Temporal profile and (c) spectrum of the shaped pulses. The red traces represent the target signals for optimum flatness derived from ISSFM calculations and the blue traces represent the experimentally optimised signals. PC: Polarisation Controller; EDFA: Erbium Doped Fibre Amplifier; OSA: Optical Spectrum Analyser.

Our experimental setup is shown in Fig. 2a. The MLL emitted 10GHz, 2ps Gaussian pulses at 1550nm. The pulses were fed to the programmable phase and amplitude filter (Finisar Waveshaper - W/S), which has a dynamic range of $35 \mathrm{~dB}$ and insertion loss of $5 \mathrm{~dB}$. The polarisation dependent loss of the W/S is $0.2 \mathrm{~dB}$ and its individual spectral features can be programmed at $1-\mathrm{GHz}$ steps, much finer than the spectral line spacing of the $10 \mathrm{GHz}$ source itself. The shaped pulses at the output of the W/S were amplified to $28 \mathrm{dBm}$ and launched to a 1-km long HNLF with a nonlinearity coefficient of $18 / \mathrm{W} / \mathrm{km}$, loss of $2.13 \mathrm{~dB} / \mathrm{km}$ and a dispersion of $-1.7 \mathrm{ps} / \mathrm{nm} / \mathrm{km}$ at $1550 \mathrm{~nm}$ with a dispersion slope of $0.0023 \mathrm{ps} / \mathrm{nm}^{2} / \mathrm{km}$. The output of the HNLF was monitored by an OSA and was fed to the ES algorithm which in turn controlled the W/S phase profile. Since our goal was to improve the flatness at the top of the generated spectra, evaluation of the $M F$ function was restricted to include contributions only from wavelengths lying within the $-5 \%$ bandwidth of the target ideal super-Gaussian spectrum $\left(S_{\text {target }}\right)$.

The red trace in Fig.2b shows the target intensity profile as calculated from the ISSFM and exhibits a pulse width of $\sim 13$ ps. In order to demonstrate the effect of our technique, we first present in Fig.3a the experimentally generated spectrum of (unshaped) Gaussian pulses of the same pulse width, as obtained at the output of the HNLF. The spectrum exhibits the familiar multitude of spectral peaks, typical of SPM in a normal dispersion fibre. Fig. $3 b$ 
presents the generated spectrum at the output of the HNLF when the transfer characteristic calculated by the ISSFM was directly loaded to the W/S. The effect of pulse pre-shaping was to reduce significantly the number of peaks on the spectrum, even though a substantial spectral lobe was developed in the middle of the spectrum. As mentioned above, a variety of factors may have contributed to the discrepancy between the generated and target spectra. It should be noted that a simple addition of linear phase profiles to the filter response did not improve substantially the flatness of the generated spectra.
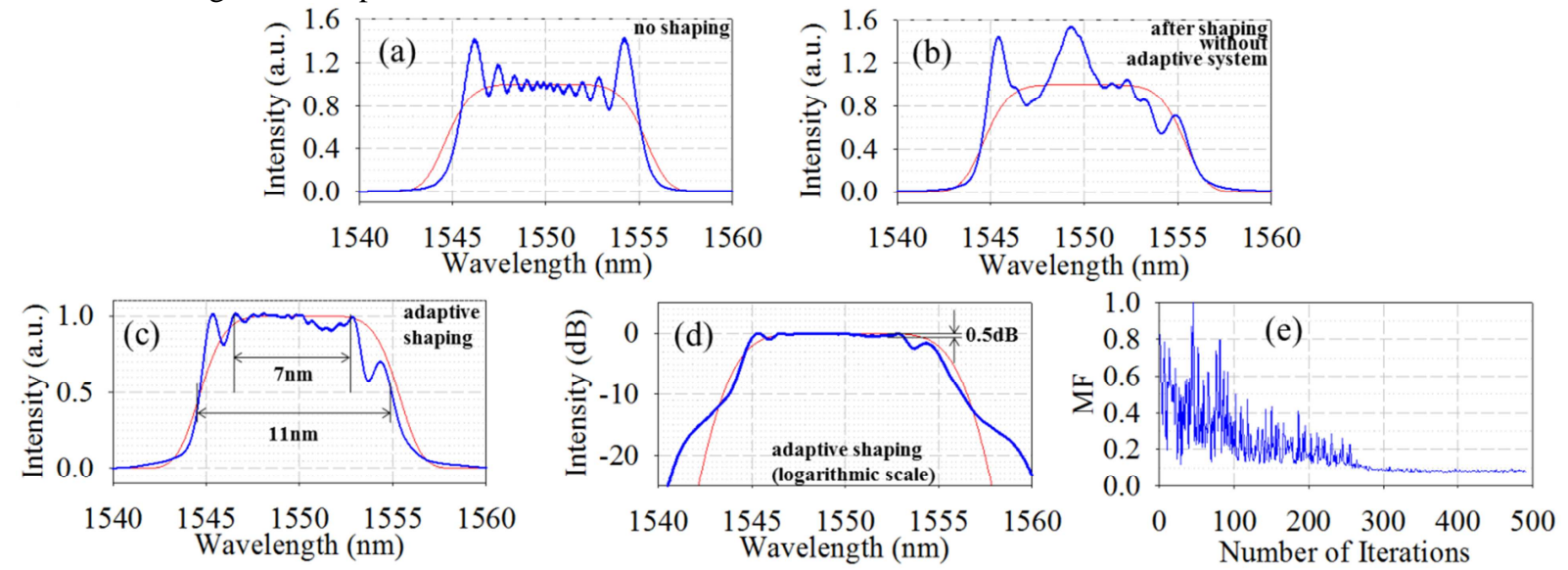

Fig. 3 SPM spectra generated from (a) 13ps Gaussian pulses, (b) the waveform calculated directly from the ISSFM and (c and d) after application of the adaptive system in linear and logarithmic scale respectively. Red traces: target spectra, blue traces: generated spectra. (e) Evolution of $M F$ value in the two-membered ES algorithm.

Fig. $3 \mathrm{c}$ shows the generated spectrum at the output of the system when adaptive shaping was in place. In Fig. 3e the $M F$ value versus the number of algorithm iterations is plotted - a ten-fold convergence to $M F=0.078$ within 300 iterations is observed. The flatness of the generated spectrum is shown to be dramatically improved exhibiting a 3$\mathrm{dB}$ bandwidth of $11 \mathrm{~nm}$ with a central region of $7 \mathrm{~nm}$ where the fluctuation is less than $0.5 \mathrm{~dB}$. The temporal and spectral profiles of the waveform yielding this spectrum are shown in Fig.2b and c (blue traces) showing very good agreement with the originally calculated waveform. Note that the spectra in Figs.3a-c are presented on a linear scale. In order to appreciate the quality of the generated spectrum, Fig. $3 \mathrm{c}$ is presented on a logarithmic scale in Fig.3d.

\section{Conclusions}

We have presented the application of an arbitrary spectral generation technique based on adaptive pulse pre-shaping and nonlinear spectral broadening in an HNLF. We have applied this technique for the nonlinear generation of flat broadband spectra and have achieved a spectrum with a $0.5-\mathrm{dB}$ bandwidth of $7 \mathrm{~nm}$. We believe that the technique can be extended to be used for the nonlinear generation of even broader spectra with arbitrary shapes.

\section{Reference}

[1] Weiner, A.M., Femtosecond pulse shaping using spatial light modulators. Review of Scientific Instruments, 2000. 71(5): p. 1929-1960.

[2] Petropoulos, P., et al., Rectangular pulse generation based on pulse reshaping using a superstructured fiber Bragg grating. Journal of Lightwave Technology, 2001. 19(5): p. 746-752.

[3] Baxter, G., et al., Highly programmable Wavelength Selective Switch based on Liquid Crystal on Silicon switching elements. 2006 Optical Fiber Communication Conference/National Fiber Optic Engineers Conference, Vols 1-6, 2006: p. 94-96.

[4] Hooper, L.E., et al., All-Normal Dispersion Photonic Crystal Fiber for Coherent Supercontinuum Generation. 2010 Conference on Lasers and Electro-Optics (CLEO), 2010: p. 2 pp.

[5] Clarke, A.M., et al., Reconfigurable Optical Pulse Generator Employing a Fourier-Domain Programmable Optical Processor. Journal of Lightwave Technology, 2010. 28(1): p. 97-103.

[6] Almeida, P.J., et al., Generation of ultra-flat SPM-broadened spectra in a highly nonlinear fiber using pulse pre-shaping in a fiber Bragg grating. 2005 Optical Fiber Communications Conference Technical Digest, 2005: p. 3 pp. Vol. 4|6 vol. (2200).

[7] Yang, X., et al., Pulse Shaping-Assitsed Nonlinear Spectral Broadening, in The European Conference on Lasers and Electro-Optics and the XIIth European Quantum Electronics Conference (CLEO®/Europe-EQEC)2011: Munich, Germany.

[8] Meshulach, D., et al., Adaptive real-time femtosecond pulse shaping. Journal of the Optical Society of America B-Optical Physics, 1998. 15(5): p. 1615-1619.

[9] Zeidler, D., et al., Evolutionary algorithms and their application to optimal control studies. Physical Review A, 2001. 64(2): p. art. no.023420 .

[10] Kashiwagi, K., et al., Highly precise optical pulse synthesis for flat spectrum supercontinuum generation with wide mode spacing. 2010 36th European Conference and Exhibition on Optical Communication (ECOC 2010), 2010: p. 3 pp.

[11] Schwefel, H.-P.P., Evolution and Optimum Seeking: The Sixth Generation 1993, New York, NY, USA: John Wiley \& Sons, Inc. 